\title{
The association between violence victimisation and common symptoms in Swedish women
}

\author{
Gunilla Krantz, Per-Olof Östergren
}

\begin{abstract}
Study objective-To investigate the association between violence and abuse suffered by women during childhood or adult life, and the manifestation of a high level of common physical and mental symptoms. Design, setting and participants-A questionnaire was sent to a random population of women, 40 to 50 years of age, living in a rural Swedish community. The response rate was 81.7 per cent (397 women). Odds ratios were used to estimate bivariate associations between the experience of violence/abuse and common symptoms. Multiple logistic regression analyses were used to test for confounding and effect modification.

Main results-The experience of violence or abuse during childhood was reported by 32.2 per cent of the women, while $\mathbf{1 5 . 6}$ per cent reported being abused as an adult. In both cases, these experiences reached statistical significance in their association with a high level of common symptoms $(O R=1.67 ; 95 \%$ CI $1.08,2.49$ and $\mathrm{OR}=2.26 ; 95 \% \mathrm{CI} 1.30,3.92$, respectively). The associations between childhood and as well adult experience of violence or abuse and common symptoms were largely independent of potential confounders such as unemployment, job strain, social support, and sense of coherence. The combined exposure to adult violence/abuse and low psychosocial coping resources, such as low social support or a low level of sense of coherence, considerably increased the odds ratio for common symptoms and a synergistic effect seemed to exist.

Conclusion-Violence or abuse experience is an important factor when considering illness manifestations in terms of common symptoms in women 40 to 50 years of age.

(F Epidemiol Community Health 2000;54:815-821)
\end{abstract}

Public Health, Box 121 33, S-402 42 Göteborg, Sweden

G Krantz

Department of Community Health Sciences, Malmö

University Hospital, Sweden

$\mathrm{P}-\mathrm{O}$ Östergren

Correspondence to: $\mathrm{Dr}$ Krantz (gunillak@nhv.se)

Accepted for publication 18 May 2000 musculoskeletal disorders and chronic pain, genitourinary disorders, and respiratory illness. ${ }^{1}$ Those who have been abused as children present a greater number of physical symptoms, as well as an increase in such psychological problems as depression, anxiety, somatisation, and low self esteem, when compared with women with no experience of ill treatment. $^{2}$ Furthermore, such women are more likely to misuse drugs and to attempt suicide. ${ }^{2}$ McCauley and colleagues showed that the number of physical symptoms increased with the severity of the violence experienced. ${ }^{3}$

Women abused during childhood report a significantly higher rate of hospitalisation and a lower estimation of their overall health. The greater the amount of abuse in childhood, the poorer the adult health profile. ${ }^{4}$

Acts of violence directed towards women is a significant public health issue both in industrialised countries and in the less developed world. ${ }^{7}$ The manifestations and forms of violence vary in different settings, and virtually wherever this issue has been researched an under-recognised burden has been unveiled. McCauley and colleagues found that in surveys of community-based populations, students, and primary care patients, from 5 to 62 per cent of the women polled revealed a history of childhood sexual abuse, while 10 to 30 per cent reported childhood physical abuse. ${ }^{3}$

Recent estimates of the global burden of disease by the World Bank suggest that, in established market economies, violence against women of reproductive age is responsible for their losing one out of every five healthy work days. $^{8}$ Comparing the industrial with the developing world, the burden of disease imposed by rape and domestic violence is roughly equivalent on a per capita basis. ${ }^{8}$

In a recently published Swedish doctoral thesis, female primary care patients aged 21 to 61 were explicitly asked if they had experienced violence or abuse. Eleven of 20 women reported such experience. ${ }^{9}$ The majority of abused women surveyed considered these episodes to have been the major reason for their subsequent pain and ill health.

Psychosocial coping resources such as social network and support, and sense of coherence have been shown to influence a person's ability to handle traumatic events and decrease the risk of negative long term effects on health. ${ }^{10}{ }^{11}$

The aim of this study was to investigate the association between violence and abuse experienced during childhood or adulthood, and the appearance of common symptoms in women, 40 to 50 years of age. Furthermore, we examined the role of psychosocial coping resources and work related factors in terms of confounding and effect modification.

\section{Methods}

POPULATION

In September 1993, a questionnaire was mailed to a random sampling of 486 women. They represented 50 per cent of the women 
between the ages of 40 and 50 in a rural community (population 13200 ) in the western part of Sweden. The response rate was 81.7 per cent, resulting in a study group of 397 women.

The circumstances of life in the community that was chosen broadly reflect those of the general population in Sweden. The principal employer is the paper industry, followed by the health professions and social services. ${ }^{12}$ Women work primarily in clerical, public service, sales, and factory jobs, while most men are engaged in business and the industrial sector.

All the data in this study were obtained through self reporting. The questionnaire contained items relating to experience of violence or abuse in childhood and in adulthood. It also inquired about socioeconomic and work related conditions, such as age, cohabitation status, educational level, income, employment, absences from work because of illness, disability pensions, psychosocial work conditions, social network/support, sense of coherence, and common symptoms.

\section{DEFINITIONS}

Common symptoms were assessed by means of the Common Symptoms in the General Population of Women (CSGP) scale, an instrument that consists of 15 physical and mental symptoms frequently found in women. ${ }^{13}$ These symptoms also correspond to what has been described in two Norwegian studies as common among women seeking care from a general practitioner. ${ }^{14}{ }^{15}$ The CSGP scale has been externally evaluated against Symptom Check List-90 $0^{16}$ and found to be a coherent instrument with a high correlation with the sub-scales mirroring somatisation, depression, and anxiety. ${ }^{13}$

The presence of each type of symptom was ranked from "almost every day" (two points) to "every week" (one point) to "every month" or "almost never, or never" (both zero points). The CSGP variable was dichotomised by the higher tertile of the distribution, so that five or more points on the scale defined a high level of symptoms. In table 1 , the 15 symptoms forming the CSGP index are displayed with prevalence rates.

A woman was considered a victim of childhood violence or abuse if she answered in the affirmative to any of the following three questions: "When you were 16 years old or younger, had you ever been exposed to any of the following: (a) occasionally been beaten by an adult? (b) regularly been beaten by an adult? (c) had an adult made sexual advances toward you?" A woman was considered a victim of adult violence or abuse if she answered affirmatively to any of the following: "During your adult years, have you ever experienced: (a) sexual advances by someone at your workplace? (b) assault and battery? (c) sexual abuse?" All responses citing either physical violence or any kind of abuse were taken together and categorised as childhood experience or adult experience, respectively. Accordingly, in the definition of adult violence and abuse, domestic violence was included but not possible to separate from other acts of violence, as respondents were not asked to differentiate between acts by partners and acts by strangers.

Cohabitation status was classified as living in the same domicile with someone, in contrast with living alone.

Educational level was based on a woman's total number of years of education and dichotomised into completed primary education (11 years) compared with higher levels of education.

The income refers to the salary obtained by the respondents (wages before taxation). It was dichotomised at the level of 10000 Swedish crowns (SEK) per month, or approximately 1200 US dollars.

Employment status was classified into two groups, employed or unemployed. In the statistical analyses, respondents with full or part time employment were combined, as the health consequences of part time employment showed more similarity to full time employment than to unemployment. ${ }^{17}$ The unemployed group included women seeking paid employment, those on leave of absence of six months or more because of illness or disability, and respondents identified as students or housewives. Sickness absence and unemployment rates for non-participants were obtained through the assistance of the regional social insurance office and the local employment office. To secure confidentiality, the data were provided only on an aggregated level.

Work related psychosocial factors were assessed by means of a Swedish version of Karasek's demand-control instrument. ${ }^{18}$ It is a well recognised instrument of good validity and reliability; it is judged a stable predictor of a person's health status. ${ }^{19}$ Participants were asked to rate their current or most recent position in terms of level of psychological job demands and level of decision latitude. Job strain denotes the combination of high psychological job demands and low decision latitude, and was defined as a job demand score above the median and a decision latitude score below the median.

To obtain social network/support indices, we used a model developed by Hanson and Östergren that quantifies social support, social anchorage, and social participation. ${ }^{20}$

Social anchorage describes the degree to which people attach themselves to formal and informal groups, as well as their feeling of belonging within those groups. Social participation describes how dynamically a person takes part in activities of formal and informal groups in society. Social support refers to the functional aspects of a person's interactions with their social network. Each of these three indices was dichotomised at the lower tertile, whereby people who scored below that level were considered to have low social support, low social anchorage, and low social participation, respectively. Both the social network/support scales and the demand-control instrument have demonstrated good validity and reliability also when applied to women. ${ }^{21} 22$

The sense of coherence (SOC) instrument developed by Antonovsky assesses three components: comprehensibility, manageability, and 
Table 1 The prevalence of the 15 symptoms forming the Common Symptoms in the General Population of women scale (CSGP) $n=397$

\begin{tabular}{lllll}
\hline & $\begin{array}{l}\text { Almost daily } \\
\text { (2 points) } \\
n \text { (\%) }\end{array}$ & $\begin{array}{l}\text { Weekly } \\
(1 \text { point) } \\
n(\%)\end{array}$ & $\begin{array}{l}\text { Monthly/never } \\
\text { (O points) } \\
n(\%)\end{array}$ \\
\hline 1 & Heartburn & $17(4.3)$ & $23(5.8)$ & $357(89.9)$ \\
2 & Stomach pain & $16(4.0)$ & $27(6.8)$ & $354(89.2)$ \\
3 & Palpitation & $11(2.8)$ & $30(7.6)$ & $356(89.7)$ \\
4 & Breathing disorder & $12(3.0)$ & $11(2.8)$ & $374(94.2)$ \\
5 & Listlessness & $15(3.8)$ & $23(5.8)$ & $359(90.4)$ \\
6 & Irritability & $28(7.1)$ & $85(21.4)$ & $284(71.5)$ \\
7 & Restlessness & $20(5.0)$ & $34(8.6)$ & $343(86.4)$ \\
8 & Nervousness/anxiety & $27(6.8)$ & $47(11.8)$ & $323(81.4)$ \\
9 & Depression & $16(4.0)$ & $25(6.3)$ & $356(89.7)$ \\
10 & Headache & $25(6.3)$ & $58(14.6)$ & $314(79.1)$ \\
11 & Tiredness & $81(20.4)$ & $114(28.7)$ & $202(50.9)$ \\
12 & Muscular tension & $74(18.6)$ & $55(13.9)$ & $268(67.5)$ \\
13 & Chest pain & $12(3.0)$ & $17(4.3)$ & $368(92.7)$ \\
14 & Low back pain & $59(14.9)$ & $28(7.1)$ & $310(78.1)$ \\
15 & Pain in the joints & $92(23.2)$ & $48(12.1)$ & $257(64.7)$ \\
\hline
\end{tabular}

meaningfulness. ${ }^{1123}$ According to his theory, the stronger the SOC, the more likely a person should be able to cope successfully with stressful situations.

The scale that Antonovsky developed to measure SOC in people originally consisted of 29 items. This scale has proved to be psychometrically sound and reliable when tested on Swedish men and women. ${ }^{24}$ In this study, we used the abbreviated version of the scale comprising 13 items. ${ }^{11}$ This shorter scale showed similar properties as the longer one when validated against the Sickness Impact Profile (SIP) scale, and it proved to have a good discriminating validity. ${ }^{25}$

Respondents were asked to choose a number from one ("never") to seven ("very often") on each of the items forming the SOC scale. The scores were added, giving a possible range of 13-91. The distribution of scores was then dichotomised at the lower tertile. Those woman who scored under that level were considered to have a low sense of coherence.

\section{STATISTICAL PROCEDURES}

Odds ratios were used to estimate the bivariate association between childhood and adult experience of violence or abuse and a high level of common symptoms.

Multiple logistic regression analysis was used wherein the strongest determinants of common symptoms were tried as potential confounding factors. However, as social support and social anchorage were relatively highly cor-

Table 2 Prevalence of violencelabuse and symptom score (CSGP index) as means and standard error

\begin{tabular}{lrrr}
\hline & & & \\
Variables & Number & $\begin{array}{l}\text { Prevalence } \\
(\%)\end{array}$ & $\begin{array}{l}\text { Symptom score } \\
\text { (CSGP) } \\
\text { Mean (SEM) }\end{array}$ \\
\hline $\begin{array}{l}\text { Childhood experience of violence or abuse: } \\
\text { None }\end{array}$ & 263 & 66.2 & $3.6(1.09)$ \\
Yes & 128 & 32.2 & $5.3(0.47)$ \\
Data missing & 6 & 1.5 & \\
Type of experience: & 109 & 27.5 & $5.0(40.4)$ \\
Occasionally beaten by adult & 13 & 3.3 & $5.2(1.36)$ \\
Sexual advances by adult & 6 & 1.5 & $11.8(3.34)$ \\
Regularly beaten by adult & 326 & 82.1 & $3.7(0.26)$ \\
Adult experience of violence or abuse: & 62 & 15.6 & $6.2(0.69)$ \\
None & 9 & 2.3 & \\
Yes & 18 & 4.7 & $4.5(0.96)$ \\
Data missing & 32 & 8.2 & $6.9(1.09)$ \\
Type of experience: & 12 & 3.1 & $7.2(1.36)$ \\
Sexual advances in the workplace & & & \\
Assault and battery & & & \\
Sexual abuse & & & \\
\hline
\end{tabular}

related it was decided to include only the social support variable. To assess the potential confounding effect regarding the relation between violence or abuse experienced during childhood and a high level of common symptoms, the variables age, employment status, social support, and SOC were entered one by one. In the next analysis the employment status variable was exchanged by the job strain variable to explore the contribution of work conditions resulting in the following variables being entered: age, job strain, social support, and SOC.

Potential confounding regarding the associations between adult experience of violence or abuse and a high level of common symptoms were assessed in the same manner.

To test for a possible effect modification of social support and sense of coherence respectively, on the relation between violence and common symptoms, a synergy index (SI) was calculated as proposed by Rothman. ${ }^{26}$ This was done by, in the first case, combining the variables assessing childhood experience of violence/abuse and social support into dummy variables so that women who had no experience of childhood violence/abuse and were not in a situation with a low social support constituted the reference category.

Consequently, the women with childhood experience of violence/abuse but with high/ medium social support, the women with no childhood experience of violence/abuse but with a low level of social support, and the women who were exposed to both factors formed the other exposure categories.

The following algorithm was used to determine effect modification using a synergy index where SI > 1 signified a synergistic effect and SI $<1$ an antagonistic effect ${ }^{26}$ :

$$
\mathrm{SI}=\frac{\mathrm{RR}(\mathrm{AB})-1}{[\mathrm{RR}(\mathrm{Ab})-1]+[\mathrm{RR}(\mathrm{aB})-1]}
$$

$\mathrm{RR}=$ risk ratio

$\mathrm{Ab}=$ exposed to one of the factors

$\mathrm{aB}=$ exposed to the other factor

$\mathrm{AB}=$ exposed to both factors

Odds ratios were used as estimates of relative risks.

This procedure was then repeated with the sense of coherence variable substituting the social support variable. In the same manner effect modification was tested regarding adult experience of violence and abuse.

The Statistical Package for the Social Sciences (SPSS) was used for all statistical purposes. ${ }^{27}$ Statistical significance was determined at the 95 per cent confidence interval (95\% CI) level.

The Ethics Committee of Gothenburg University has given its approval to this research project.

\section{Results}

Of the 486 women contacted for this study, 397 women (81.7 per cent) responded. Respondents were vocationally active to a greater degree than non-respondents (80.6 versus 62.9 per cent). Non-respondents were more often sick 
Table 3 Association between demographic and psychosocial variables and a high level of common symptoms (CSGP index), presented as crude odds ratios (OR) and 95 per cent confidence intervals (95\% CI)

\begin{tabular}{|c|c|c|c|}
\hline Variables & Number & $\begin{array}{l}\text { High level of } \\
\text { symptoms, \% }\end{array}$ & Crude OR $(95 \% \mathrm{CI})$ \\
\hline \multicolumn{4}{|l|}{ Cohabitation status: } \\
\hline Cohabiting & 337 & 33.0 & 1 (reference) \\
\hline Living alone & 57 & 42.1 & $1.48(0.80,2.74)$ \\
\hline \multicolumn{4}{|l|}{ Educational level: } \\
\hline$>11$ years & 147 & 30.2 & 1 (reference) \\
\hline$\leqslant 11$ years & 233 & 37.7 & $1.45(0.93,2.25)$ \\
\hline \multicolumn{4}{|l|}{ Monthly income: } \\
\hline$>10000 \mathrm{SEK}$ & 206 & 33.0 & 1 (reference) \\
\hline$\leqslant 10000 \mathrm{SEK}$ & 167 & 36.5 & $1.17(0.76,1.79)$ \\
\hline \multicolumn{4}{|l|}{ Employment status: } \\
\hline Employed & 320 & 29.4 & 1 (reference) \\
\hline Unemployed & 77 & 53.2 & $2.74(1.65,4.55)$ \\
\hline \multicolumn{4}{|l|}{ Work related psychosocial factors: } \\
\hline No job strain & 233 & 25.3 & 1 (reference) \\
\hline Job strain & 109 & 49.5 & $2.90(1.80,4.67)$ \\
\hline \multicolumn{4}{|l|}{ Social network/support indices: } \\
\hline \multicolumn{4}{|l|}{ Social support: } \\
\hline $\mathrm{High} /$ medium & 303 & 28.4 & 1 (reference) \\
\hline Low & 94 & 52.1 & $2.75(1.71,4.42)$ \\
\hline \multicolumn{4}{|l|}{ Social anchorage: } \\
\hline $\mathrm{High} /$ medium & 303 & 28.1 & 1 (reference) \\
\hline Low & 94 & 53.2 & $2.91(1.81,4.69)$ \\
\hline \multicolumn{4}{|l|}{ Social participation: } \\
\hline $\mathrm{High} /$ medium & 261 & 29.9 & 1 (reference) \\
\hline Low & 136 & 41.9 & $1.69(1.10,2.61)$ \\
\hline \multicolumn{4}{|l|}{ Sense of coherence: } \\
\hline High/medium & 267 & 20.1 & 1 (reference) \\
\hline Low & 130 & 60.8 & $5.84(3.69,9.24)$ \\
\hline \multicolumn{4}{|c|}{ Childhood experience of violence or abuse: } \\
\hline None & 263 & 30.4 & 1 (reference) \\
\hline Yes & 128 & 42.2 & $1.67(1.08,2.59)$ \\
\hline \multicolumn{4}{|l|}{ Type of experience: } \\
\hline Occasionally beaten by adult & 109 & 40.4 & $1.55(0.97,2.46)$ \\
\hline Sexual advances by adult & 13 & 38.5 & $1.43(0.45,4.51)$ \\
\hline Regularly beaten by adult & 6 & 83.3 & $11.44(1.32,99.48)$ \\
\hline \multicolumn{4}{|l|}{ Adult experience of violence or abuse: } \\
\hline None & 326 & 30.7 & 1 (reference) \\
\hline Yes & 62 & 50.0 & $2.26(1.30,3.92)$ \\
\hline \multicolumn{4}{|l|}{ Type of experience: } \\
\hline Sexual advances in the workplace & 18 & 38.9 & $1.44(0.54,3.82)$ \\
\hline Assault and battery & 32 & 53.1 & $2.56(1.23,5.33)$ \\
\hline Sexual abuse & 12 & 58.3 & $3.16(0.98,10.21)$ \\
\hline
\end{tabular}

listed (7.9 compared with 2.0 per cent) or were more prone to have obtained disability pensions (13.5 compared with 5.0 per cent).

Among the 15 symptoms compiled into the CSGP index, the most prevalent symptoms among the respondents were pain in the joints, tiredness, muscular tension, low back pain, headache and irritability, as presented in table 1.

Prevalence rates concerning exposure to violence and abuse are displayed in table 2. It was found that 128 women (32.2 per cent) reported exposure to childhood violence or abuse, while 62 women (15.6 per cent) reported experience of violence or abuse as adults.

Furthermore, we found that 40 women (10 per cent) had been subjected to violence or abuse both as children and as adults. The highest mean symptom score (11.8) was found among women who reported having been beaten regularly by an adult during childhood;
KEY POINTS

- In a random sample of women, 40 and 50 years of age, living in a rural community in Sweden, 32.2 per cent had experienced violence or abuse during childhood, while 15.6 per cent reported adult experience of violence or abuse.

- Having experienced violence or abuse in adulthood more than doubled the risk of a high level of common physical and mental symptoms among the women in the sample. This association was largely independent of potential confounders such as unemployment, job strain, social network/support, and sense of coherence.

- Exposure to violence or abuse during childhood increased the odds ratio for a high level of common symptoms by about 60 per cent, which was statistically significant.

- Adequate social support and a high level of sense of coherence seemed to have a protective effect on the association between violence experienced at adult age and a high level of common symptoms.

- Exposure to violence or abuse, either during childhood or adulthood, is a serious trauma that continues to exert influence on a woman's health as common symptoms in her later life.

the second highest mean symptom score was 7.2 for adults who had experienced sexual abuse.

The associations between demographic and psychosocial variables and a high level of common symptoms are presented as crude odds ratios with 95 per cent confidence intervals in table 3. Women who experienced violence or abuse as a child or as an adult had a significantly higher odds ratio for a high level of common symptoms, as compared with women with no such experience $(\mathrm{OR}=1.67 ; 95 \% \mathrm{CI}$ $1.08,2.59$ and $\mathrm{OR}=2.26 ; 1.30,3.92$, respectively).

Women who had been beaten regularly in childhood had the highest odds ratio for a high level of common symptoms $(\mathrm{OR}=11.44 ; 1.32$, 99.48). This was followed by adult victims of sexual abuse $(\mathrm{OR}=3.16 ; 0.98,10.21)$ and victims of assault and battery $(\mathrm{OR}=2.56 ; 1.23$, 5.33).

When further examining the relation between experience of violence during childhood and its association with common symptoms in a multivariate analysis, we found that the odds ratio remained at the same level when controlling for age, employment status, social support,

Table 4 Association between childhood experience of violencelabuse and a high level of common symptoms, presented as adjusted odds ratios and confidence intervals (95\% CI; $n=397)$

\begin{tabular}{lllll}
\hline & Model 1 & Model 2 & Model 3 & Model 4 \\
\hline Childhood experience (yes/no) & $1.67(1.07,2.61)$ & $1.68(1.07,2.64)$ & $1.60(1.01,2.55)$ & $1.64(1.00,2.68)$ \\
Employed (no/yes) & & $2.61(1.55,4.41)$ & $2.43(1.43,4.14)$ & $2.40(1.36,4.25)$ \\
Social support (low/high) & & & $2.28(1.39,3.76)$ & $1.61(0.93,2.77)$ \\
SOC (low/high) & & & $4.87(2.96,7.99)$ \\
\hline
\end{tabular}


Table 5 Association between adult experience of violencelabuse and a high level of common symptoms, presented as adjusted odds ratios and confidence intervals (95\% CI; $n=397$ )

\begin{tabular}{|c|c|c|c|c|}
\hline & Model 1 & Model 2 & Model 3 & Model 4 \\
\hline Adult experience (yes/no) & $2.40(1.36,4.24)$ & $2.05(1.14,3.69)$ & $2.06(1.14,3.74)$ & $1.92(1.02,3.62)$ \\
\hline Employed (no/yes) & & $2.52(1.48,4.31)$ & $2.33(1.35,4.01)$ & $2.28(1.28,4.06)$ \\
\hline Social support (low/high) & & & $2.25(1.36,3.74)$ & $1.65(0.95,2.85)$ \\
\hline SOC (low/high) & & & & $4.63(2.81,7.62)$ \\
\hline
\end{tabular}

and sense of coherence, $\mathrm{OR}=1.64(1.00,2.68)$, as presented in table 4 . When employment status was exchanged for job strain in the model, the odds ratio remained at the same level all through the analysis, though failing to reach significance in the full model when SOC was added, $\mathrm{OR}=1.61(0.94,2.75)$ (not in table).

In the same manner, experience of adult violence and abuse and its association with common symptoms was tested in a multivariate model, controlling for the same possible confounding variables. We then found that the odds ratio declined somewhat when employment status was entered but it remained statistically significant in the full model, OR 1.92 $(1.02,3.62)$ as displayed in table 5 . When substituting the unemployment variable for the job strain variable a similar pattern emerged with an odds ratio for adult experience of violence or abuse of $2.13(1.08,4.22)$ in the full model (not in table).

Finally, we investigated how the association between violence/abuse experience and common symptoms was affected when there was a concurrent exposure to low social support or to a low level of sense of coherence (table 6). Being exposed to violence/abuse during childhood and as well low social support only moderately increased the odds ratio for a high level of common symptoms (adjusted for age, cohabitation status, educational level and income), OR 3.71 (1.74, 7.91). To test for effect modification, we calculated the synergy index that was 1.10 , thus indicating only a very marginal synergistic relation.

The impact of a simultaneously low SOC on the association between violence experienced during childhood and common symptoms was considerable, OR=9.98 (4.58, 21.75). The SI

Table 6 Test for effect modification of the association between childhood or adult experience of violencelabuse and common symptoms regarding exposure to psychosocial coping resources, presented as adjusted odds ratios (OR) with confidence intervals $(95 \%$ CI), and synergy index (SI). $n=397$

\begin{tabular}{llll}
\hline Variables & OR & $95 \%$ CI & \multirow{2}{*}{$S I$} \\
\hline Childhood experience of violence/abuse and social support: & & & \\
No childhood experience/high or medium social support & 1 & reference & \\
Childhood experience/high or medium social support & 1.51 & $0.86,2.66$ & \\
No childhood experience/low social support & 2.94 & $1.49,5.81$ & \multirow{2}{*}{1.10} \\
Childhood experience/low social support & 3.71 & $1.74,7.91$ & \\
Childhood experience of violence/abuse and sense of coherence: & & & \\
No childhood experience/high or medium SOC & 1 & reference & \\
Childhood experience/high or medium SOC & 1.30 & $0.67,2.52$ & \\
No childhood experience/low SOC & 5.02 & $2.73,9.25$ & \multirow{2}{*}{ Childhood experience/low SOC } \\
Adult experience of violence/abuse and social support: & 9.98 & $4.58,21.75$ & \\
No adult experience/high or medium social support & 1 & reference & \\
Adult experience/high or medium social support & 2.17 & $1.06,4.44$ & \\
No adult experience/low social support & 2.81 & $1.58,5.00$ & \multirow{2}{*}{2.40} \\
Adult experience/low social support & 8.17 & $2.43,27.43$ & \\
Adult experience of violence/abuse and sense of coherence: & & & \\
No adult experience/high or medium SOC & 1 & reference & \\
Adult experience/high or medium SOC & 2.44 & $1.09,5.44$ & \\
No adult experience/low SOC & 5.49 & $3.17,9.51$ & \multirow{2}{*}{1.71} \\
Adult experience/low SOC & 11.13 & $4.02,30.85$ & \\
\hline
\end{tabular}

was 2.08, indicating a possible synergistic relation.

When investigating the association between with adult experience of violence/abuse and common symptoms with concurrent exposure to low social support or low SOC, we found increased odds ratios, OR $8.17(2.43,27.43)$ and OR $11.13(4.01,31.11)$ respectively, compared with being exposed to adult violence/ abuse only. The respective SI was 2.40 and 1.71 , suggesting a possible synergistic relation in both cases.

\section{Discussion}

This study found that in a random sample of women between the ages of 40 and 50, living in a rural community in Sweden, 32.2 per cent had experienced violence or abuse during childhood, and 15.6 per cent as adults. Furthermore, we found that exposure to violence or abuse in adulthood more than doubled the risk of a high level of common symptoms, and this risk decreased only moderately when controlling for a number of potentially confounding variables. Exposure to violence or abuse during childhood also increased the risk of a high level of common symptoms by about 60 per cent, which was statistically significant.

When investigating if effect modification was at hand we found some indication on this. Low sense of coherence showed a possible synergistic relation with both childhood and adult experience of violence or abuse, and low social support seemed to act synergistically with adult exposure to violence/abuse regarding the association with a high level of common symptoms. In other words, this suggests that the association between violence victimisation and common symptoms would be attenuated in the presence of high/medium social support or high/medium level of sense of coherence.

METHODOLOGICAL CONSIDERATIONS

In this study there was a stronger association between experience of adult violence and common symptoms than between experience of childhood violence and common symptoms. This might imply that time is a moderating factor. However, the acts of violence inquired about during childhood was possibly phrased less harshly than the acts of violence at adult age, which also ought to be considered.

The instrument used to estimate violence experienced by the respondents yielded prevalence rates in our study group, which correspond well with findings in other studies. ${ }^{328}$ This leads us to believe that our instrument was reasonably sensitive for the purpose intended. 
It is widely assumed that experience of violence or abuse might be underestimated because of mere underreporting, as a woman's experience of childhood or adult violence/ abuse is a sensitive piece of information, which she might hesitate to reveal. Consequently, there is a risk of selection bias in this study as we believe that women who had been abused probably had a greater reluctance to participate. Combined with the general tendency of a better health status among participants, this might have resulted in more exposed cases among non-participants. ${ }^{29}$ If so, this would lead to an underestimation of the true association between violence victimisation and common symptoms in our sample.

There was little evidence of confounding when analysing the relations between either childhood or adult experience of violence/ abuse and common symptoms. The odds ratios remained statistically significant and of almost the same strength when strong predictors of common symptoms, such as unemployment, job strain, low social support or low SOC were entered into the models. An exception was the relation between experience of childhood violence or abuse and common symptoms, which failed to reach statistical significance in the full model with the job strain variable present. It seems though as if acts of violence or abuse, experienced either during childhood or at adult age, are independent risk factors, each associated with a high level of common symptoms in women aged 40 to 50 years.

In our testing of effect modification we found possible synergistic relations but we are aware that these data need to be interpreted with caution, as the study population is rather small. However, the findings suggest that social support and sense of coherence have a protective effect, which is of importance when planning preventive activities.

In conclusion, our aim with this research was to increase the knowledge for establishing effective preventive and treatment procedures. The idea was to focus on women with symptoms shown to be possible manifestations of stress $^{13}$ and to avoid well defined medical conditions, which could be caused by a number of other specific determinants. No assessment of such medical conditions that could have contributed to a high level of common symptoms or an assessment of the influence of treatment has been made as that was beyond the scope of this study.

Our findings could have some important implications for future public health and social interventions directed at women with ongoing or past experience of violence or abuse.

Well known psychological effects of victimisation are loss of self esteem, increased negative views of self and decreased feelings of personal control. A supportive social network of family, friends and professionals seems most important in enabling women to regain some of this and could contribute to reducing stress. Support needs to be easily accessible at the local level and could be offered as self help support groups, women's shelters, counselling activities, multi-professional teams.
Within this context, it is worth noting that Antonovsky was convinced that comprehensibility, manageability and meaningfulness, which form the sense of coherence concept, could be strengthened in tailor-made interventions and he proposed that the salutogenic model could be viewed as a guide within health promotion work. ${ }^{30}$ Swedish researchers, in collaboration with Antonovsky, further elaborated this and found that those with a strong sense of coherence also had more general motivation and less somatic and psychic anxiety as well as less hostility than those who scored low on the SOC scale. ${ }^{24}$ This kind of knowledge might be valuable when planning individualised caring or health promoting interventions.

We believe that all women presenting in primary health care with multiple symptoms should be asked about experiences of violence or abuse, as it most often goes unrecognised and unreported. Training on how to handle the health consequences of violence and abuse against women should be included in postgraduate medical curriculums. In collaboration with women patients and other professionals, doctors could assist in seeking comprehensive plans to reduce this multifaceted public health problem.

Funding: this study was supported by grants from the National Institute of Public Health and the Swedish Council for Social Research.

Conflicts of interest: none.

1 Leserman J, Li Z, Drossman DA, et al. Selected symptoms associated with sexual and physical abuse history among female patients with gastrointestinal disorders: the impact on subsequent health care visits. Psychol Med 1998;28:41725.

2 McCauley J, Kern DE, Kolodner K, et al. Clinical haracteristics of women with a history of childhood abuse: unhealed wounds. $\mathcal{F A M A}$ 1997;227:1362-8.

3 McCauley J, Kern DE, Kolodner K, et al. Relation of low-severity violence to women's health. $\mathcal{f}$ Gen Intern Med 1998;13:687-91.

4 Moeller TP, Bachmann GA, Moeller JR. The combined effects of physical, sexual and emotional abuse during childhood: long-term health consequences for women. Child Abuse and Neglect 1993;17:623-40.

5 Golding JM, Cooper ML, George LK. Sexual assault history and health perceptions: seven general population history and health perceptions: seven

6 Smith PH, Gittelman DK. Psychological consequences of Smith PH, Gittelman DK. Psychological consequences of battering: implications for women's
practice. $N C$ Med $\mathcal{F}$ 1994;55:434-9.

7 Heise LL, Raikes A, Watts CH, et al. Violence against women: a neglected public health issue in less developed countries. Soc Sci Med 1994;39:1165-79.

8 Heise LL, Pitanguy J, Germain A. Violence against women: the hidden health burden. Washington DC: World Bank, 1994.

9 Hamberg K, Johansson EE, Lindgren G. "I was always on guard"-an exploration of woman abuse in a group of women with musculoskeletal pain. Fam Pract 1999;16:23844.

10 Testa M, Miller BA, Downs WR, et al. The moderating impact of social support following childhood sexual abuse. Violence and Victims 1992;7:173-86.

11 Antonovsky A. Unraveling the mystery of health. San Francisco: Jossey-Bass, 1987.

12 Statistics Sweden. The equality programme. Örebro: SCBtryck, 1993.

13 Krantz G, Östergren P-O. Women's health: do common symptoms in women mirror general distress or specific disease entities? Scand F Public Health 1999;27:311-17.

14 Brage S, Tellnes G. ICPC i allmenpraksis (ICPC in general practice). Tidskr Nor Laegeforening 1992;112:2656-9.

15 Nylenna $M$. Why do our patients see us? Scand $\dot{f}$ Prim Health Care 1985;3:155-62.

16 Derogatis LR, Cleary PA. Confirmation of the dimensional structure of the SCL-90: a study in construct validation. $\mathcal{F}$ Clin Psychol 1977;33:981-9.

17 Verbrugge LM. Multiple roles and physical health of women and men. F Health Soc Behav 1983;24:16-30.

18 Karasek R, Theorell T. Healthy work, stress productivity and the reconstruction of working life. New York: Basic Books, 1990.

19 Kristensen TS. Sickness absence and work strain among Danish slaughterhouse workers: an analysis of absence Danish slaughterhouse workers: an analysis of absence
from work regarded as coping behaviour. Soc Sci Med 1991;32:15-27. 
20 Hanson BS, Östergren P-O. Different social network and social support characteristics, nervous problems and insomnia: theoretical and methodological aspects on some results from the population study "Men born in 1914 in Malmö, Sweden". Soc Sci Med 1987:25:849-59.

21 Hanson BS, Östergren P-O, Elmståhl S, et al. Reliability and validity assessments of measures of social networks, social support and control-results from the Malmö shoulde and neck study. Scand $\mathcal{F}$ Soc Med 1997;25:249-57.

22 Theorell T. On cardiovascular health in women: results from epidemiological and psychosocial studies in Sweden. In: Frankenhaeuser M, Lundberg U, Chesney M, eds. Women, work and health: stress and opportunities. New York: Plenum Press, 1991:187-204

23 Antonovsky A. Health, stress and coping. San Francisco: Jossey-Bass, 1979

24 Langius A, Björvell $\mathrm{H}$, Antonovsky A. The sense of coherence concept and its relation to personality traits in
Swedish samples. Scand $\mathcal{F}$ Caring Sci 1991;6:165-71.
25 Langius A, Björvell $\mathrm{H}$. Coping ability and functional status in a Swedish population sample. Scand $\mathcal{F}$ Caring Sci 1993; $7: 3-10$

26 Rothman KJ. Modern epidemiology. Boston: Little, Brown, 1986.

27 Norusis M. Statistical package for the social sciences. Professional statistics release 6.1 (SPSS 6.1). Chicago: SPSS, 1994.

28 Folkhälsorapport 1997 (National public health report 1997). Stockholm: Socialstyrelsen (National board of health and welfare), 1997. Report no 1997:18

29 Krantz G, Östergren P-O. Common symptoms in middle aged women: their relation to employment status, psychosocial work conditions and social support in a Swedish setting. I Epidemiol Community Health 2000;54:192-9.

30 Antonovsky A. The salutogenic model as a theory to guide health promotion. Health Promotion International 1996;11: $11-8$. 